\title{
Review of: "Estimating and evaluating the rice cluster distribution uniformity with UAV-based images"
}

\author{
Edison Pignaton de Freitas $^{1}$ \\ 1 Universidade Federal do Rio Grande do Sul
}

Potential competing interests: The author(s) declared that no potential competing interests exist.

I consider the authors present a very good proposal for a UAV-based precision agriculture application on rice fields. The paper is very well-written and it provides very consistent and convincing results.

I have just some minor suggestions for enhancing the paper before publication:

1. I suggest the authors to avoid using sentences in the first person (our), prefer using sentences in the third person instead.

2. I think the authors could highlight the contributions of the paper at the end of the introduction.

3. The literature review can be enhanced. I think the authors could enrich the discussion including more citations to other related works presenting UAV-based precision agriculture applications with image processing support, such as:

A UAV Guidance System Using Crop Row Detection and Line Follower Algorithms. Journal of Intelligent Robotic Systems 97, 605-621 (2020). https://doi.org/10.1007/s10846-019-01006-0

4. The concluding section is weak. It can be more elaborated. Moreover, I suggest the authors provide a separate paragraph at the end of the conclusion specifically for future works' discussion, way more elaborated than the sentence about this that is there. 